\title{
Further Evaluation of Howland Integrals
}

\author{
By Chih-Bing Ling
}

Abstract. The purpose of this paper is to further evaluate two Howland integrals to 25D when their index is an even integer.

In a previous paper [1], Ling and Lin evaluated the following two Howland integrals to $25 \mathrm{D}$ when their index $k$ is an odd integer:

$$
\begin{array}{ll}
I_{k}=\frac{1}{2(k !)} \int_{0}^{\infty} \frac{w^{k} d w}{\sinh w \pm w} \quad(k \geqslant 1) \\
I_{k}^{*} \quad(k \geqslant 3)
\end{array}
$$

Recently, the author encountered a need of highly precise values of these two integrals when their index is an even integer. This occurs in the evaluation of certain allied integrals of a similar nature. The computation incurs rapid loss of significant figures, although the desired accuracy is of a lower degree. In order to meet the need, the two integrals are further evaluated in this paper to 25D when their index is an even integer.

The following expansions shown in the previous paper hold for any index $k$, even or odd:

$$
\begin{aligned}
& I_{k}=1 \mp \frac{q_{2}(k)}{2^{k+2}}+\frac{q_{3}(k)}{3^{k+3}} \mp \frac{q_{4}(k)}{4^{k+4}}+\cdots, \quad(k \geqslant 1) \\
& (k \geqslant 3),
\end{aligned}
$$

where, for $n \geqslant 0$,

$$
q_{2 n+1}(k)=\sum_{m=0}^{n}(-1)^{n+m}\left(\begin{array}{c}
2 m+k \\
k
\end{array}\right) \frac{(n+m) !}{(n-m) !} 2^{2 m}(2 n+1)^{2 n-2 m}
$$

$$
q_{2 n+2}(k)=\sum_{m=0}^{n}(-1)^{n+m}\left(\begin{array}{c}
2 m+k+1 \\
k
\end{array}\right) \frac{(n+m+1) !}{(n-m) !} 2^{2 m+1}(2 n+2)^{2 n-2 m} .
$$

The series in (2) converges rather slowly when the index is a small integer. For instance, to attain an accuracy of $25 \mathrm{D}, 50$ terms of the series are needed when $k \leqslant 20$; but only ten terms are needed when $k \geqslant 33$.

When the index is an even integer, the following two pairs of expansions are derived in a similar manner by using the method described in the previous paper, which is a modification of Plana's method:

Received August 15, 1977.

AMS (MOS) subject classifications (1970). Primary 41A30, $65 \mathrm{A05}$.

Key words and phrases. Evaluation of Howland integrals. 


$$
\begin{aligned}
& I_{2 k}=\frac{1}{(2 k) !}\left[\frac{\alpha}{\pi} \sum_{n=1}^{\infty} \frac{(n \alpha)^{2 k} \operatorname{Si}(n \pi)}{\sinh n \alpha+n \alpha}\right. \\
& \left.+1 / 2 \operatorname{Re} \sum_{m=1}^{\infty} \frac{z_{m}^{2 k}\left\{E_{1}\left(a_{m}\right) \exp \left(a_{m}\right)+E^{*}\left(a_{m}\right) \exp \left(-a_{m}\right)\right\}}{\cosh ^{2}\left(z_{m} / 2\right) \sinh a_{m}}\right], \\
& I_{2 k}=\frac{1}{(2 k) !}\left[\frac{\alpha}{\pi} \sum_{n=0}^{\infty} \frac{(n \alpha+1 / 2 \alpha)^{2 k} \operatorname{Si}(n \pi+1 / 2 \pi)}{\sinh (n+1 / 2) \alpha+(n+1 / 2) \alpha}\right. \\
& \left.+1 / 2 \operatorname{Re} \sum_{m=1}^{\infty} \frac{z_{m}^{2 k}\left\{E_{1}\left(a_{m}\right) \exp \left(a_{m}\right)-E^{*}\left(a_{m}\right) \exp \left(-a_{m}\right)\right\}}{\cosh ^{2}\left(z_{m} / 2\right) \cosh a_{m}}\right] \text {, }
\end{aligned}
$$

(4)

$$
\begin{aligned}
& I_{2 k}^{*}=\frac{1}{(2 k) !}\left[\frac{\alpha}{\pi} \sum_{n=1}^{\infty} \frac{(n \alpha)^{2 k} \operatorname{Si}(n \pi)}{\sinh n \alpha-n \alpha}\right. \\
& \left.+1 / 2 \operatorname{Re} \sum_{m=1}^{\infty} \frac{z_{m}^{* 2 k}\left\{E_{1}\left(a_{m}^{*}\right) \exp \left(a_{m}^{*}\right)+E^{*}\left(a_{m}^{*}\right) \exp \left(-a_{m}^{*}\right)\right\}}{\sinh ^{2}\left(z_{m}^{*} / 2\right) \sinh a_{m}^{*}}\right], \\
& I_{2 k}^{*}=\frac{1}{(2 k) !}\left[\frac{\alpha}{\pi} \sum_{n=0}^{\infty} \frac{(n \alpha+1 / 2 \alpha)^{2 k} \operatorname{Si}(n \pi+1 / 2 \pi)}{\sinh (n+1 / 2) \alpha-(n+1 / 2) \alpha}\right. \\
& \left.+1 / 2 \operatorname{Re} \sum_{m=1}^{\infty} \frac{z_{m}^{* 2 k}\left\{E_{1}\left(a_{m}^{*}\right) \exp \left(a_{m}^{*}\right)-E^{*}\left(a_{m}^{*}\right) \exp \left(-a_{m}^{*}\right)\right\}}{\sinh ^{2}\left(z_{m}^{*} / 2\right) \cosh a_{m}^{*}}\right],
\end{aligned}
$$

where

$$
a_{m}=-\pi i z_{m} / \alpha, \quad a_{m}^{*}=-\pi i z_{m}^{*} / \alpha
$$

The first pair is valid for $k \geqslant 1$ and the second pair for $k \geqslant 2$. $\alpha$ is a positive constant, which can be fixed to suit our convenience. $z_{m}$ and $z_{m}^{*}$ are the $m$ th complex zeros of ( $\sinh z \pm z$ ), respectively, in the first quadrant of the $z$ plane. Si is a sine integral and $E_{1}$ and $E^{*}$ are exponential integrals [2] defined by

$$
\begin{gathered}
\operatorname{Si}(a)=\int_{0}^{a} \frac{\sin t}{t} d t, \\
E_{1}(a)=\int_{a}^{\infty} \frac{e^{-t}}{t} d t, \\
E^{*}(a)=-f_{-a}^{\infty} \frac{e^{-t}}{t} d t, \quad(\operatorname{Re}[a]>0) .
\end{gathered}
$$

The last integral is a Cauchy principal value. They are introduced into the expansions through the integrals: 


$$
\begin{aligned}
\int_{0}^{\infty} \frac{\sin (\pi t / \alpha) d t}{n^{2} \alpha^{2}-t^{2}} & =-\frac{(-1)^{n}}{n \alpha} \operatorname{Si}(n \pi) \\
\int_{0}^{\infty} \frac{t \cos (\pi t / \alpha) d t}{(n \alpha+1 / 2 \alpha)^{2}-t^{2}} & =(-1)^{n} \operatorname{Si}(n \pi+1 / 2 \pi) \\
\int_{0}^{\infty} \frac{\sin (\pi t / \alpha) d t}{t^{2}+a^{2}} & =\frac{1}{2 a}\left\{E_{1}\left(\frac{\pi a}{\alpha}\right) \exp \left(\frac{\pi a}{\alpha}\right)+E^{*}\left(\frac{\pi a}{\alpha}\right) \exp \left(-\frac{\pi a}{\alpha}\right)\right\} \\
\int_{0}^{\infty} \frac{t \cos (\pi t / \alpha) d t}{t^{2}+a^{2}} & =1 / 2\left\{E_{1}\left(\frac{\pi a}{\alpha}\right) \exp \left(\frac{\pi a}{\alpha}\right)-E^{*}\left(\frac{\pi a}{\alpha}\right) \exp \left(-\frac{\pi a}{\alpha}\right)\right\}
\end{aligned}
$$

Although the preceding expansions are more complicated than the analogous ones for an odd index, yet their properties are essentially alike. Each expansion consists of two series. Each first series converges more rapidly when $\alpha$ is large and each second series when $\alpha$ is small. In the computation, $\alpha$ is likewise taken as unity. The four first series involve $\operatorname{Si}(n \pi / 2)$ and the four second series $z_{m}, z_{m}^{*}, E_{1}$ and $E^{*}$. The values of $\operatorname{Si}(n \pi / 2)$ have been tabulated by Ling and $\operatorname{Lin}$ [3] to 25D for $n=1(1) 200$, together with a factor $2 / \pi$. Further values can be generated easily whenever needed. The complex zeros $z_{m}$ and $z_{m}^{*}$ have been tabulated by Ling and Cheng [4] to 11D for both real and imaginary parts. Their accuracy can be improved readily by using the Newton-Raphson method. The following series are suitable for computing $E_{1}$ and $E^{*}$ when $|\arg a|<\pi$ :

$$
E_{1}(a)=-\gamma-\ln a+e^{-a} \sum_{n=1}^{\infty}\left(1+\frac{1}{2}+\frac{1}{3}+\cdots+\frac{1}{n}\right) \frac{a^{n}}{n !}
$$

$$
E^{*}(a)=\gamma+\ln a+\sum_{n=1}^{\infty} \frac{a^{n}}{n(n !)},
$$

where $\gamma$ is Euler constant. Or, when $|a|$ is large, they are given by the following asymptotic series:

$$
\begin{aligned}
& E_{1}(a) \sim \frac{e^{-a}}{a}\left(1-\frac{1}{a}+\frac{2 !}{a^{2}}-\frac{3 !}{a^{3}}+\cdots\right), \\
& E^{*}(a) \sim \frac{e^{a}}{a}\left(1+\frac{1}{a}+\frac{2 !}{a^{2}}+\frac{3 !}{a^{3}}+\cdots\right),
\end{aligned}
$$

so that

$$
\begin{aligned}
& E_{1}(a) e^{a}+E^{*}(a) e^{-a} \sim \frac{2}{a}\left(1+\frac{2 !}{a^{2}}+\frac{4 !}{a^{4}}+\cdots\right), \\
& E_{1}(a) e^{a}-E^{*}(a) e^{-a} \sim-\frac{2}{a^{2}}\left(1+\frac{3 !}{a^{2}}+\frac{5 !}{a^{4}}+\cdots\right) .
\end{aligned}
$$

Note that in the present computation the real part of $a_{m}$ or $a_{m}^{*}$ is always positive and greater than the imaginary part numerically. 


\section{TABLE 1}

Howland integrals $I_{2 k}$ and $I_{2 k}^{*}$

\begin{tabular}{|c|c|c|c|c|c|c|c|c|c|c|}
\hline $2 k$ & \multicolumn{5}{|c|}{$I_{2 k}$} & \multicolumn{5}{|c|}{$I_{2 k}^{*}$} \\
\hline 2 & 0.76784 & 74391 & 33919 & 04735 & 95563 & & $\infty$ & & & \\
\hline 4 & 0.88350 & 68065 & 08692 & 59048 & 39136 & 1.35329 & 41151 & 70484 & 00917 & 07709 \\
\hline 6 & 0.95419 & 15618 & 26139 & 06398 & 92330 & 1.07672 & 97636 & 74217 & 12721 & 31355 \\
\hline 8 & 0.98412 & 41801 & 48424 & 61430 & 92995 & 1.02053 & 76000 & 64959 & 33956 & 30213 \\
\hline 10 & 0.99492 & 24398 & 53445 & 37390 & 51763 & 1.00578 & 48422 & 24523 & 50460 & 14460 \\
\hline 12 & 0.99845 & 99579 & 47832 & 38304 & 10890 & 1.00164 & 49762 & 53520 & 38988 & 10881 \\
\hline 14 & 0.99954 & 93055 & 62626 & 17685 & 89104 & 1.00046 & 58410 & 12174 & 41784 & 75036 \\
\hline 16 & 0.99987 & 13214 & 26371 & 77772 & 61970 & 1.00013 & 08088 & 09455 & 33470 & 89835 \\
\hline 18 & 0.99996 & 39030 & 69165 & 32295 & 73410 & 1.00003 & 63897 & 74380 & 02428 & 04253 \\
\hline 20 & 0.99999 & 00058 & 51207 & 35354 & 88793 & 1.00001 & 00336 & 28030 & 38688 & 48032 \\
\hline 22 & 0.99999 & 72607 & 78826 & 41420 & 23605 & 1.00000 & 27444 & 55935 & 02623 & 90687 \\
\hline 24 & 0.99999 & 92552 & 82148 & 05839 & 47506 & 1.00000 & 07454 & 02213 & 09431 & 30333 \\
\hline 26 & 0.99999 & 97988 & 78365 & 74311 & 66056 & 1.00000 & 02012 & 10025 & 40280 & 85053 \\
\hline 28 & 0.99999 & 99459 & 88927 & 60932 & 42466 & 1.00000 & 00540 & 22369 & 86530 & 35487 \\
\hline 30 & 0.99999 & 99855 & 65214 & 61417 & 58450 & 1.00000 & 00144 & 36216 & 21557 & 16743 \\
\hline 32 & 0.99999 & 99961 & 58384 & 19634 & 14263 & 1.00000 & 00038 & 41795 & 57087 & 48030 \\
\hline 34 & 0.99999 & 99989 & 81377 & 14198 & 99581 & 1.00000 & 00010 & 18645 & 28389 & 31179 \\
\hline 36 & 0.99999 & 99997 & 30790 & 95789 & 84203 & 1.00000 & 00002 & 69211 & 82207 & 76456 \\
\hline 38 & 0.99999 & 99999 & 29059 & 58456 & 74027 & 1.00000 & 00000 & 70940 & 75809 & 71445 \\
\hline 40 & 0.99999 & 99999 & 81355 & 37962 & 27125 & 1.00000 & 00000 & 18644 & 66239 & 92477 \\
\hline 42 & 0.99999 & 99999 & 95111 & 46854 & 25576 & 1.00000 & 00000 & 04888 & 53658 & 68928 \\
\hline 44 & 0.99999 & 99999 & 98721 & 02338 & 73696 & 000 & 00000 & 01278 & 97723 & 61257 \\
\hline 46 & 0.99999 & 99999 & 99666 & 04495 & 193 & 1.0 & 00000 & 00333 & 95512 & 35557 \\
\hline 48 & 0.99999 & 99999 & 99912 & 95851 & 94238 & 1.00000 & 00000 & 00087 & 04148 & 96852 \\
\hline 50 & 0.99999 & 99999 & 99977 & 35145 & 08454 & 1.00000 & 00000 & 00022 & 64855 & 02501 \\
\hline 52 & 99999 & 99999 & 99994 & 11581 & 80352 & 00 & 00000 & 00005 & 88418 & 20962 \\
\hline 54 & 0.99999 & 99999 & 99998 & 47344 & 33490 & 000 & 00000 & 00001 & 52655 & 66667 \\
\hline 56 & 0.99999 & 99999 & 99999 & 60448 & 30484 & 1.00000 & 00000 & 00000 & 39551 & 69535 \\
\hline 58 & 0.99999 & 99999 & 99999 & 89765 & 13150 & 000 & 00000 & 00000 & 10234 & 86852 \\
\hline 60 & 0.99999 & 99999 & 99999 & 97354 & 54670 & 1.00000 & 00000 & 00000 & 02645 & 45330 \\
\hline 62 & 0.99999 & 99999 & 99999 & 99316 & 952 & & 00000 & 00000 & 00683 & 04737 \\
\hline 64 & 0.99999 & 99999 & 99999 & 99823 & 817 & & 00000 & 00000 & 00176 & 18285 \\
\hline 66 & 0.99999 & 99999 & 99999 & 99954 & 59903 & 1.00000 & 00000 & 00000 & 00045 & 40097 \\
\hline 68 & 0.99999 & 99999 & 99999 & 99988 & 31095 & 000 & 00000 & 00000 & 00011 & 68905 \\
\hline 70 & 0.99999 & 99999 & 99999 & 99996 & 99303 & 1.00000 & 00000 & 00000 & 00003 & 00697 \\
\hline 72 & 0.99999 & 99999 & 99999 & 99999 & 227 & & 00000 & 00000 & 00000 & 77292 \\
\hline 74 & 0.99999 & 99999 & 99999 & 99999 & 80148 & & 00000 & 00000 & 00000 & 19852 \\
\hline 76 & 0.99999 & 99999 & 99999 & 99999 & 94905 & 1.00000 & 00000 & 00000 & 00000 & 05095 \\
\hline 78 & 0.99999 & 99999 & 99999 & 99999 & 98693 & 1.00000 & 00000 & 00000 & 00000 & 01307 \\
\hline 80 & 0.99999 & 99999 & 99999 & 99999 & 99665 & 1.00000 & 00000 & 00000 & 00000 & 00335 \\
\hline 82 & 0 & 99999 & 99999 & 999 & 999 & 1.00000 & 00000 & 00000 & 00000 & 00086 \\
\hline 84 & 0.99999 & 99999 & 99999 & 99999 & 99978 & 1.00000 & 00000 & 00000 & 00000 & 00022 \\
\hline 86 & 0.99999 & 99999 & 99999 & 99999 & 99994 & 1.00000 & 00000 & 00000 & 00000 & 00006 \\
\hline 88 & 0.99999 & 99999 & 99999 & 99999 & 99999 & 1.00000 & 00000 & 00000 & 00000 & 00001 \\
\hline 90 & 1.00000 & 00000 & 00000 & 00000 & 00000 & 1.00000 & 00000 & 00000 & 00000 & 00000 \\
\hline
\end{tabular}


To attain an accuracy of 25D for the two integrals with $\alpha=1,190$ terms in the first series and three terms in the second series are needed when $2 k \leqslant 60$, or 130 terms in the first series and three terms in the second series when $2 k \leqslant 30$. If the value of $\alpha$ is doubled, the number of terms needed in the first series is halved but that in the second series is doubled.

The resulting values of the two integrals are computed from (4) for $2 k \leqslant 60$ and from (2) for $2 k \geqslant 56$. The overlapped values are used for checking purposes. The values computed from each pair of expansions in (4) are in agreement as they ought to be. The following relations may be used as an additional check:

$$
\sum_{k=1}^{\infty} k\left(1-I_{2 k}\right)=I_{2}-\frac{1}{16}, \quad \sum_{k=2}^{\infty} k\left(I_{2 k}^{*}-1\right)=\frac{17}{16} .
$$

Comparison was made with Nelson's $18 \mathrm{D}$ values [5]. It revealed no discrepancy in Nelson's results.

The computation was carried out on an IBM 370 Computer with extended precision; and the 25D results for $2 k$ from 2 to 90 , inclusive, appear in Table 1 .

In conclusion, it may be mentioned that, as an alternate method of evaluation, one might attempt to use the Gregory-Newton interpolation formula to evaluate the two integrals, because the values for odd index have been tabulated. However, it was found that by this formula adequate precision could not be obtained, particularly for small even index, owing to the behavior of the integrals.

Department of Mathematics

Virginia Polytechnic Institute and State University

Blacksburg, Virginia 24061

1. C. B. LING \& J. LIN, "A new method of evaluation of Howland integrals," Math. Comp., v. 25, 1971, pp. 331-337. MR 45 \#4603.

2. A. ERDÉLYI, W. MAGNUS, F. OBERHETTINGER \& F. G. TRICOMI, Higher Transcendental Functions, Vol. II, McGraw-Hill, New York, 1953, pp. 143-145. MR 15, 419.

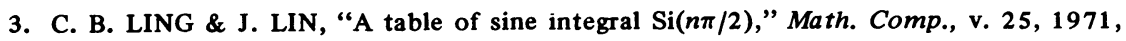
RMT 21, p. 402.

4. C. B. LING \& F. H. CHENG, "Stresses in a semi-infinite strip," (appendix), Internat. J. Engrg. Sci., v. 5, 1967, p. 169.

5. C. W. NELSON, "New tables of Howland's and related integrals," Math. Comp., v. 15, 1961, pp. 12-18. MR 22 \#10203. 